\title{
Philosophiques
}

\section{Le privilège culturel et la politique de la reconnaissance}

\section{Mette Hjort}

Volume 23, numéro 1, printemps 1996

Critères esthétiques et métamorphoses du beau

URI : https://id.erudit.org/iderudit/027364ar

DOI : https://doi.org/10.7202/027364ar

Aller au sommaire du numéro

Éditeur(s)

Société de philosophie du Québec

ISSN

0316-2923 (imprimé)

1492-1391 (numérique)

Découvrir la revue

Citer cet article

Hjort, M. (1996). Le privilège culturel et la politique de la reconnaissance. Philosophiques, 23(1), 47-55. https://doi.org/10.7202/027364ar

\section{Résumé de l'article}

Les théories post-colonialistes nous ont montré à quel point l'absence d'une reconnaissance de la valeur de certaines formes culturelles produit des effets nuisibles. Ces théories ignorent pourtant la nature asymétrique des relations régissant l'interaction entre des cultures mineures et des cultures majeures, c'est-à-dire entre les grandes et les petites nations. Par conséquent, la nécessité d'une politique de la reconnaissance spécifique aux petites nations privilégiées n'est pas reconnue. Afin d'établir ce point, j'examine quelques aspects de la production cinématographique danoise. J'essaie aussi de montrer que les cinéastes appartenant à des petites nations privilégiées doivent adopter une stratégie précise de la reconnaissance.
Ce document est protégé par la loi sur le droit d'auteur. L'utilisation des services d'Érudit (y compris la reproduction) est assujettie à sa politique d'utilisation que vous pouvez consulter en ligne.

https://apropos.erudit.org/fr/usagers/politique-dutilisation/ 
PHILOSOPHIgUES, VOL. XXIII, N 1, PRINTEMPS 1996, P. 47-55

\title{
IE PRIVIIĖGE CUITUREL ET LA POLITIQUE DE LA RECONNAISSANCE
}

\author{
PAR \\ Metie HJort
}

\begin{abstract}
RÉSUMÉ : Les théories post-colonialistes nous ont montré à quel point l'absence d'une reconnaissance de la valeur de certaines formes culturelles produit des effets nuisibles. Ces théories ignorent pourtant la nature asymétrique des relations régissant l'interaction entre des cultures mineures et des cultures majeures, c'est-à-dire entre les grandes et les petites nations. Par conséquent, la nécessité d'une politique de la reconnaissance spécifique aux petites nations privilégièes n'est pas reconnue. Afin d'établir ce point, j'examine quelques aspects de la production cinématographique danoise. J'essaie aussi de montrer que les cinéastes appartenant à des petites nations privilégiées doivent adopter une stratégie précise de la reconnaissance.
\end{abstract}

ABSTRACT: Influential postcolonial theories have successfully shown that the failure to recognize the value of certain cultural forms inflicts a form of harm. What these theories fail to note is that small, prosperous nations also require a politics of recognition as a result of the asymmetrical nature of relations governing major and minor cultures. To prove this point I consider aspects of contemporary Danish cinematic production. I attempt to identify the strategy for recognition that is best suited to filmmakers belonging to small, but prosperous nations.

Il est banal de dire que le cinéma comporte une dimension expressive et communicative. Après tout, les films ne sont-ils pas destinés à ètre vus par un public ? Ce qui toutefois demeure inconnu, c'est la nature de la relation concrète qui existe entre un film donné et ses spectateurs ou ses publics. Dans ce texte j'examine la relation entre le film et ses publics dans un contexte particulier de la production cinématographique, un contexte où la réflexion sur cette relation fondamentale est urgente en raison des hiérarchies qui y prévalent. Plus précisément, j'examinerai certaines méthodes qu'emploient les cinéastes danois lorsqu'ils cherchent à inscrire leurs œuvres dans un corpus de films reconnus sur le plan international. Je m'intéresse particulièrement à la création d'un corpus dominant de films internationaux et aux facteurs qui font que des citoyens de petites nations veulent que leurs films en fassent partie. 
Plusieurs aspects de la production cinématographique danoise et de son accueil par le public sont déterminés par les hiérarchies culturelles existantes. Il faut donc examiner les traits qui figurent dans certains jugements de valeur portant sur des films, de méme que les raisons qui permettent d'attribuer aux œuvres une valeur supérieure ou inférieure. Les cultures, comme nous le savons, peuvent être classifiées et hiérarchisées de plusieurs façons. Pierre Bourdieu, par exemple, parle de goûts "populaires ", "moyens " et "légitimes ", évoquant ainsi un ensemble de distinctions culturelles qui prévalent dans les nations où les agents utilisent stratégiquement les jugements esthétiques afin de construire et négocier des hierrarchies sociales et économiques ${ }^{1}$. D'autre part, Gilles Deleuze et Félix Guattari tracent une distinction entre les cultures majeures et les cultures mineures; ils imaginent les traits d'une "littérature mineure " capable de subvertir la culture ou le langage dominant d'un État-nation donné ${ }^{2}$. Bien que Deleuze et Guattari limitent leur analyse au contexte culturel de la Tchécoslovaquie de Franz Kafka, la distinction entre culture majeure et culture mineure peut facilement être mise à profit dans l'analyse d'une politique de la culture sur le plan international.

Des jugements comparatifs sur des caractéristiques intrinsèques des œuvres - comme leurs qualités esthétiques - ne peuvent pas déterminer la valeur relative des produits culturels, et parfois les jugements esthétiques ne jouent aucun rôle dans la construction des hiêrarchies de valeur. Le fait que certains objets culturels soient reconnus dans la sphère culturelle internationale dépend en grande partie de facteurs économiques, démographiques et géopolitiques, quoique les membres des cultures dominantes y voient malheureusement l'indice d'une supériorité culturelle, et mẻme la justification d'une démarcation entre cultures mineures et majeures. Dans les cas extrêmes, la prédominance culturelle devient une sorte de monologue intense capable d'écarter de la sphère publique des formes variées d'expressions culturelles.

Quand on fait partie d'une culture mineure, on se heurte à la nature problématique des différents publics puisqu'un statut inférieur dans une hiérarchie des cultures est directement relié à des attitudes d'indifférence ou de mépris du public. Il semble que dans le cas des cultures mineures, le manque d'intérêt vienne non pas d'un mais de plusieurs publics, desquels un petit nombre seulement sont situés au-delà des frontières de l'État-nation. Dans des petits pays comme le Danemark, les cinéastes doivent faire face à des publics locaux qui préférent à l'art "sérieux " des films comiques où des blondes folâtrent dans des tas de foin. Bien que cette situation ne soit pas particulière aux petites nations, elle est exacerbée par d'autres facteurs qui eux le sont. Plus précisément, l'impérialisme culturel dans lequel s'engagent les grands États-nations tend à monopoliser non seulement les marchés mais les publics. Par exemple, pendant les négociations du GATT, un politicien français a fait remarquer qu'il

1. Voir Pierre Bourdieu, La distinction Critique sociale du jugement, Paris, Éditions de Minuit (1979).

2. Voir Gilles Deleuze \& Félix Guattari, * Gu'est-ce qu'une littérature mineure * dans Kafka Pour une littérature mineure, Paris, Editions de Minuit (1975). 
est présentement impossible de voir un film européen dans une salle de cinéma publique au Portugal. La saturation des marchés implique un processus d'acculturation, ce que les Français appellent, dans un esprit critique, la mondialisation ${ }^{3}$. Un public qui doit se rabattre sur des films comme Working Girl et le Parc jurassique développe des goûts qui jurent avec les modes locaux d'expressions culturelles.

La nature "monologique "de certains types de publics internationaux, dominés par un petit nombre de nations ou de cultures majeures, rend particulièrement difficile la possibilité, pour les membres des petites nations, de s'exprimer de façon authentiquement personnelle. Les publics internationaux sont souvent fortement unilingues; en faire partie présuppose une maitrise de la langue favorisée par la culture dominante. Tandis que les membres des cultures mineures doivent être multilingues s'ils veulent faire partie d'un public international, les membres des cultures majeures n'ont qu'à exploiter leur langue maternelle. Dans certains contextes, les traces d'une spécificité nationale-les accents, l'utilisation de langues étrangères, et même les sous-titres - seront perçues comme autant de différences étranges et déplaisantes par rapport à ce qui est dominant et apparemment naturel.

Les difficultés que rencontre toute entreprise qui vise à trouver un public national et international pour les films danois ne sont aucunement récentes et particulières à cette nation européenne. Les Danois, cependant, ressentent le problème des publics de façon très dramatique, car la situation actuelle peut etre contrastée avec celle d'un àge d'or révolu du cinéma danois. Comme on le sait, la sonorisation - de mème qu'une certaine politique de la langue favorisant seulement certaines langues - a sapé les bases de ce qui était jusqu'à l'aube de la Première Guerre mondiale une florissante industrie danoise du film muet, reconnue sur le plan international pour les qualités techniques, réalistes et érotiques de ses produits ${ }^{4}$.

Toute réflexion sur la nécessité de promouvoir le cinéma danois soulève inévitablement la question des publics. Par exemple, un argument assez répandu veut que celui qui met l'accent sur la spécificité culturelle vise un double but. Il ne faut pas, dit-on, seulement créer une culture nationale authentique, mais favoriser les conditions d'une reconnaissance internationale de certains produits culturels. En parlant de son film Pelle le Conquérant, Bille August établit un lien direct entre la spécificité culturelle d'un film et son attrait sur le plan international : "Ce qui me plait beaucoup dans ce film c'est son cōté franchement nordique. Je pense qu'en faisant des films qui gardent une forte identité nationale nous pouvons en faire des films internationaux. Quand nous essayons d'imiter, par exemple, les films d'action américains nous nous retrouvons avec des produits qui n'ont aucune espèce d'identité $e^{5}$. "Carl Dreyer était du même avis lorsqu'il parlait d'un film sur Hans Christian Andersen qu'il aimerait réaliser : "C'est une erreur de penser qu'on améliore l'attrait d'un film à l'étranger en en faisant un produit de manufacture international. Au

3. En français dans le texte. [Note du traducteur]

4. Voir Ron Mottram. The Danish Cinema Before Dreyer, Metuchen, N. J. et Londres, Scarecrow Press (1988).

5. Cité par Jytte Jensen, "Bille's Feast ", The Village Voice (7 juin 1988), p. 59. 
contraire, le film sera d'autant plus apprécié qu'il nous montrera tels que nous sommes et sans nous abaisser devant les goūts qui prévalent à l'étranger ${ }^{6}$. "C'est seulement si nous montrons les traits qui nous distinguent que nous pouvons espérer être reconnus par les autres. Nous y perdons de trois façons si nous essayons simplement de nous conformer aux standards du goūt international. Procéder ainsi signifie un rejet d'un certain engagement en faveur d'une expression authentique et une tentative d'être reconnu pour de mauvaises raisons. D'autre part, la probabilité qu'un créateur qui est bien intégré dans une culture mineure puisse comprendre et maitriser les standards en question est plutôt faible. Enfin, il est assez rare qu'on se réjouisse lorsqu'un artiste se montre capable de faire passer ses ouvres pour des produits de la culture dominante.

Le fait que les discussions sur la spécificité nationale mènent à la question des publics internationaux montre que Thomas Guback laisse échapper quelque chose d'essentiel lorsqu'il décrit le but d'un cinêma national : "Le but, dit-il, n'est pas d'entrer en compétition avec les films qui s'adressent à des publics internationaux, mais d'utiliser le cinéma pour présenter ou clarifier les problèmes, la vie et les aspirations des gens". "Le but, comme Guback l'affirme, est en effet celui de permettre à l'identité d'une culture nationale de s'exprimer authentiquement. Cependant, il est impérieux que l'identité soit reconnue par les publics à l'extérieur de l'État-nation. Notre tâche devient donc celle de préciser pourquoi il est si crucial d'être reconnu internationalement. Parallèlement, il importe aussi de déterminer comment la reconnaissance internationale peut être obtenue sans contrecarrer le désir de s'exprimer authentiquement.

Il a èté suggéré que le penchant internationaliste du cinéma national danois puisse être partiellement expliqué par le rōle que l'État a joué dans le processus de la production cinématographique ${ }^{8}$. En tant que principal producteur des films danois, l'État chercherait à augmenter le nombre de publics pour ses produits. Bien que le financement se fasse sous forme de prêts aux cinéastes et aux producteurs, les conditions de paiement sont très souples. En effet, la plupart des films danois ne réussissent même pas à atteindre le niveau de profits à partir duquel l'État exige un remboursement. Il est sans doute intéressant de réfléchir sur le rōle que joue l'État dans le processus d'internationalisation des films, mais il semble peu

6. Carl Dreyer, "New Roads for the Danish Film " dans Dreyer in Double Reflection, sous la dir. de Donald Skoller, New York, Dutton (1973), p. 8182 .

7. Thomas Guback, " Hollywood's International Market " dans Tino Balio, The American FUm Industry, Madison, University of Wisconsin Press (1976). p. 406.

8. "80emes danske film fra A til $Z$ ", Lene Nordin, Kosmorama, 33 (1987). p. 40. Pour Andrew Higson la recherche du profit est une des principales raisons d'être du cinéma national : "L'histoire du cinéma national [...] est en fait l'histoire d'une entreprise qui cherche à bien s'intëgrer au marché pour maximiser ses profits tout en permettant à la culture nationale de s'affirmer. Dans ce domaine, la politique de cinèma national peut ètre réduite à une stratégie de marketing, c'est-à-dire à une tentative de mettre en marché la diversitê comme quelque chose qui peut offrir une expérience coherente et unique. Andrew Higson, "The Concept of National Cinema ", Screen, 30 (1989), p. 37-38. 
probable que le désir des membres de l'industrie cinématographique de faire circuler certains films dans la sphère publique internationale puisse ètre expliqué adéquatement de cette façon. Il faut plutôt rechercher cette explication dans ce que Charles Taylor a appelé la "politique de la reconnaissance "(the politics of recognition) ${ }^{9}$.

Un des points importants soulevés par Taylor veut que l'identité se développe de façon " dialogique ", sinon en conversation, du moins en relation avec les autres. Par exemple, la perception qu'on a de soi est très influencée par les valeurs morales du groupe auquel on appartient. Pourtant, comme le précise Taylor, les agents se tournent aussi vers ces "autres donneurs de sens ${ }^{10}$ "(significant others), c'està-dire vers des groupes qui n'ont pas la mème identité collective. Aussi pour expliquer cette orientation vers l'autre, Taylor parle-t-il d'un besoin humain d'ètre reconnu. Ce besoin, soutient-il, est la principale motivation à la base des discours de plus en plus influents des groupes dominés ou minoritaires, qu'il s'agisse de groupes de Noirs, de féministes, de gaies ou de lesbiennes. Quand nous parvenons à comprendre le besoin d'ètre reconnus qu'éprouvent ces agents, il devient clair que le fait de ne pas reconnaitre ou d'apprécier certaines identités individuelles ou collectives puisse représenter une forme de violence.

La reconnaissance, affirme Taylor, s'inscrit dans une transition entre la période pré-moderne et la période moderne. Dans les contextes où les identités sont socialement dérivées et sont fixes et stables, la reconnaissance se fait automatiquement. Cependant, avec l'effondrement de la hiérarchie de l'ètre, l'identité individuelle est générée intérieurement plutôt que socialement, ce qui favorise les conditions dans lesquelles " la tentative d'être reconnu peut échouer $^{11}$. "De ce point de vue, la nature problématique de la reconnaissance provient directement de certains aspects de la modernité.

Certains agents subissent les effets nuisibles causés par le fait de ne pas être reconnus pour ce qu'ils sont, ou de ne pas ètre reconnus du tout. Ce sont ces agents qui auront tendance à politiser la question de la reconnaissance. Selon Taylor, la politique de la reconnaissance pratiquée par les groupes minoritaires met à profit deux courants de la pensée moderne. D'une part, un discours de l'authenticité, que l'on peut faire remonter au romantisme, et selon lequel c'est la valeur d'un soi généré intérieurement, d'un soi authentique, qui doit être reconnue. Si nous devons cette idée d'authenticité aux romantiques, c'est à des penseurs des Lumières, comme Kant, que, d'autre part, nous devons les notions modernes d'égalité et de dignité. Ce sont ces notions qui sont à la base des discours sur les droits de la personne, lesquels tablent sur l'uniformisation des droits et des titres [entitlements]. Quand ces deux courants de la pensée moderne se combinent, comme cela se fait dans la politique de la reconnaissance, il ne reste plus qu'à affirmer que l'individu a le droit

9. Voir " La politique de reconnaissance "dans Charles Taylor et Amy Gutmann, Multiculturalisme: différence et démocratie, traduit de l'anglais par Denis-Armand Canal, Paris, Aubier (1994), p. 41-99.

10. Ibid., p. 50.

11. Ibid., p. 53. 
de demander que son mode particulier d'expression personnelle soit reconnu comme ayant au moins la même valeur que les autres formes d'expressions personnelles. Le critère d'universalisabilité qui régit le discours des droits égaux s'applique donc aussi au domaine de l'expression personnelle. Taylor cherche à convaincre le lecteur de la nature problématique de cette tendance à joindre ensemble les questions de dignité et d'authenticité, mais ce thème déborde la présente discussion.

Les discours post-colonialistes et multiculturalistes qui proviennent de l'Amérique du Nord ont été très efficaces en promouvant une politique de la reconnaissance, en raison de la grande valorisation qu'on y fait des cultures issues de groupes sans pouvoir économique et politique. L'exigence de reconnaissance est donc, en Amérique du Nord, explicitement liée à un projet d'accessibilité au pouvoir politique. Il est intéressant de souligner que l'accent qui est mis sur le lien entre les pouvoirs culturel et politique dans les théories nordaméricaines ne permet pas de bien voir le besoin d'une politique de la reconnaissance dans des contextes où les pouvoirs économique et politique sont répartis de façon plus égale parmi les citoyens. Ces théories ne peuvent saisir aisément les aspirations multiculturalistes des citoyens qui appartiennent à des nations où l'on ne retrouve pas la diversité ethnique qui est caractëristique de l'Amérique du Nord. Dans le cas du Danemark, par exemple, les privilèges économiques et sociaux contribuent très peu à réduire la marginalité culturelle des Danois dans des contextes internationaux. Ce que les théories contemporaines négligent lorsqu'elles traitent des liens entre les cultures majeures et mineures, c'est la nécessité d'avoir une politique de la reconnaissance qui prenne en compte les publics internationaux, et non uniquement les publics nationaux.

Comment les cinéastes qui appartiennent à des cultures mineures peuvent-ils alors gagner la faveur d'un public international ? Une des stratégies peut consister à se conformer aux goùts internationaux. tout en interprétant la spécificité culturelle comme un obstacle à surmonter. Lars von Trier, par exemple, montre peu ou aucun attachement à la culture danoise dans des films comme Elément $d u$ crime et Europa ${ }^{12}$. Ses personnages parlent l'américain, l'anglais ou l'allemand plutôt que le danois et leurs actions sont mises en scène d'une manière qui ressuscite des images qui font penser aux films de Sternberg, Hitchcock, Welles et Tarkovski, de même qu'à certaines bandes dessinées européennes et américaines. Certes, l'on pourrait faire valoir que von Trier efface les traces d'identité nationale pour mieux soulever des questions qui touchent l'autonomie des identités collectives en Europe. L'absence de spécificité culturelle, dans ces conditions, n'est pas l'indice d'une indifférence aux problèmes d'identité. Mais si le but ici consiste à clarifier la nature d'une politique de la reconnaissance propre aux cultures mineures, il faut alors considérer une toute autre stratégie qui permette de s'assurer la faveur des publics internationaux.

12. On comprend pourquoi Daniel Sauvaget affirme que " Le Danemark est décidément un pays trop petit pour Lars von Trier *. Daniel Sauvaget, "Lars von Trier ou les délices de l'ambiguité ", La Revue du Cinéma, 476 (1991), p. 69 . 
Je pense en particulier à une stratégie du levier (leveraging strategy) par laquelle certains éléments de nature internationale sont employés afin de rendre possible la récognition de la culture danoise à l'êtranger. Cette stratégie, je crois, constitue un des aspects de ce que Benjamin Lee appelle l'"écriture trans-référentielle "(crosswriting) et de ce que l'on pourrait appeler le "film trans-référentiel * (cross-filming). L'emploi du terme "trans " (cross), dans le présent contexte, fait bien ressortir la façon dont certains cineastes orientent consciemment leurs films vers des publics ayant des goùts différents et, dans une certaine mesure, incompatibles. Dans le but de mieux comprendre comment cette double orientation peut servir la politique de la reconnaissance d'une culture mineure, il est nécessaire de se pencher sur ce qu'on peut appeler les caractères opaque, traduisible et international de certains éléments cinématographiques. Plus précisément, il s'agit de voir comment la distance causée par certains de ces élements peut ètre comblée par d'autres. Il sera utile de se référer, ce faisant, à deux films de Bille August, un cinéaste rompu aux avantages et désavantages du " film trans-référentiel *.

On peut dire qu'un élément cinématographique est opaque lorsqu'il est si ancré dans un imaginaire national donné que les membres d'un public international ne peuvent pas comprendre son sens sans l'aide d'un informateur indigène. Prenons, par exemple, dans un des premiers films de Bille August, Twist and Shout, la séquence d'événements qui évoque la ville de Svendborg, au Danemark. Dans ce film, l'action découle en partie de la relation amoureuse que vivent deux adolescents, Bjørn et Anna. Au tout début de leur liaison, Anna annonce qu'elle sera absente pour quelques jours. La réaction désespérée de Bjørn devient encore plus comique aux yeux du public danois quand Anna précise qu'elle ira à Svendborg, pas tellement loin de Copenhague, mais que les habitants de la capitale considèrent comme un bled. Le film montre toute l'anxiété que provoque chez Bjørn la séparation. C'est ainsi, par exemple, qu'il imagine des fermiers réunis dans une grange au plus fort de l'hiver pour tirer un peu de chaleur d'un strip-tease que leur offre la belle Anna. Les membres des publics internationaux ne manqueront pas de remarquer l'importance que les personnages de ce film attribuent à un endroit appelé "Svendborg ". Ce dont ils ne pourront prendre la pleine mesure, cependant, c'est l'usage dont fait August de la distance qui sépare les cultures cosmopolitaine et provinciale afin de créer une série d'effets que les publics locaux trouveront particulièrement drōles.

La situation est tout autre dans le cas des éléments traduisibles. Bien que ces éléments possèdent ici une couleur typiquement danoise, leur spécificité culturelle n'empéchera pas les membres des publics étrangers de saisir le sens des scènes où ils sont présents. Dans Pelle le Conquérant, par exemple, Lasse présente à plusieurs reprises sa propre conception d'une belle vie, qui comprend le café au lit les dimanches et du rôti de porc avec raisins - au lieu du hareng - la veille de Noël. Même si les membres des publics étrangers ne mangent ni du rôti de porc avec raisins ni du hareng la veille de Noël, la signification que prennent ces aliments est accessible à quiconque peut distinguer entre le temps de fête et le temps quotidien. En d'autres mots, les processus de la traduction 
interculturelle fonctionnent quand certaines catégories de l'expérience sociale sont partagées par des cultures quí, à d'autres égards, accusent d'importantes différences.

Tout en ayant des éléments cinématographiques opaques et traduisibles, plusieurs films danois - par exemple, Pelle le Conquérant de Bille August, Le festin de Babette de Gabriel Axel et Gertrud de Carl Dreyer - ont aussi une certaine dimension internationale. Le roman de Martin Andersen Nexø dont Pelle le Conquérant est tiré n'est pas seulement un classique de la littérature danoise, mais une ouvre qui a été canonisée par le mouvement socialiste international. Le Pelle de Nexø a été traduit en plusieurs langues et connut une grande diffusion en feuilleton au début du XX siècle. On dit mème que Lenine en a recommandé la traduction en russe après avoir lu le troisième volume dans l'Humanité, l'organe du parti communiste français ${ }^{13}$.

Les personnages centraux, Pelle et Erik, rèvent d'èmigrer en Amérique, pays d'espoir et de renouveau. Ainsi Pelle évoque les vagues d'émigration qui unissent le Danemark à d'autres nations et, en particulier, aux États-Unis. D'ailleurs August dit que d'une certaine manière l'Amérique est un élément constitutif des différentes identités européennes:

Mais il ne faut pas perdre de vue que les États-Unis sont un creuset dans lequel toutes les nations européennes sont venues se fondre. Ce qui fait que les différentes cultures ont été intégrées de façon plus ou moins subtile. Nous sommes tous un peu chez nous en Amérique ${ }^{14}$.

Il y a une supposition qui sous-tend le Pelle de Bille August et qui, si elle est juste, aide ce film à s'assurer la faveur d'un public international : tout comme certaines conceptions de l'Amérique ont façonné les différentes identités européennes, les visions de l'Europe ont contribué à la construction d'une identité américaine $e^{15}$. Les éléments internationaux présents dans Pelle aident à concevoir le Danemark comme un pays auquel peuvent, d'une certaine façon, s'identifier les membres des publics américains. Cette identification ne se limite pas aux seuls Américains de descendance danoise ou scandinave, puisque à travers la dimension internationaliste du film le Danemark apparaît comme le symbole du pays d'origine. Les conditions épouvantables qui ont amené Erik et Pelle à rèver de l'Amérique, certes, ont fort bien pu exister au Danemark, mais elles existaient ailleurs aussi.

Mais comment les trois éléments discutés ci-dessus se rapportent-ils à la question des publics d'une culture mineure et à celle de la politique de la reconnaissance, telle que pratiquée par les citoyens des petites nations? Bien que les trois éléments soient

13. Palle Schantz Lauridsen, "Fra Brønshoj til Bornholm: Pelle Erobreren er blevet en kraftfuld og stor film ", Kosmorama, 182 (1987), p. 9.

14. Cité dans Yves Alion, "Bille August : entretien ", La Revue du Cinéma, 443 (1988), p. 43.

15. Cette affirmation est facilement confirmée par les statistiques sur l'émigration qui indiquent, par exemple, que 300000 Danois se sont installés en Amérique du Nord entre 1870 et 1914. Voir Stewart Oakley, A Short History of Denmark, New York et Washington, Praeger Publishers (1972), p. 195. 
nécessaires pour séduire les publics nationaux, c'est la qualité opaque des films danois qui est déterminante. Plus précisément, l'opacité cinématographique produit une sorte d'effet de miroir : les spectateurs locaux se voient dans un film qui met en scène le Danois aussi bien que l'humour et les pratiques proprement danois. Par contre, l'interêt des publics internationaux pour un film n'est pas suscité par la perception d'une différence ou d'une spécificité culturelle, mais plutôt par des éléments déjà internationalisés. Ces derniers deviennent en quelque sorte le levier qui permet à des formes variées de spécificités culturelles d'atteindre les publics internationaux et d'ètre reconnues d'eux.

Le rōle principal que joue la stratégie du levier dans l'internationalisation des films danois est l'indice d'importantes différences entre une politique de la reconnaissance, motivée en partie par un désir de rectifier une injuste distribution des pouvoirs politique et économique, et une politique d'affirmation, issue essentiellement d'une indifférence à l'égard de la production culturelle des petites nations. Mettre l'accent de façon explicite sur la différence culturelle ne peut réussir comme stratégie de reconnaissance que si la spécificité en question est liée, aux yeux de tous, à des injustices politiques et économiques qu'une société se doit, en théorie ou en pratique, d'éliminer. Au contraire, la stratégie du levier devient nécessaire quand la différence culturelle apparait dans un contexte où existent la prospérité économique et le bon fonctionnement d'une démocratie politique, comme c'est le cas au Danemark. Remplacer la stratégie du levier et la médiation par un appel au sens de justice du public reviendrait à présenter les Danois comme les victimes d'une indifférence des publics étrangers à l'égard de leur culture. Bien que cette indifférence constitue une forme de préjudice, il faut aussi tenir compte du fait que la plupart des Danois jouissent d'une bonne prospérité. Autrement dit, les Danois font de bien mauvaises victimes sur la scène internationale.

J'ai centré mon propos ici sur ce que je considère comme le moyen le plus efficace de produire une culture cinématographique nationale capable de gagner la faveur d'un public international. Cette stratégie a déjà cours, mais devrait probablement ètre plus répandue et mieux comprise. Bien sûr, cette stratégie a ses propres limites. Par exemple, il se peut fort bien que certains aspects importants d'une culture nationale ne puissent tout simplement pas ètre liés aux éléments internationaux qui servent de levier. Si c'est le cas, comme je m'en doute, alors il serait imprudent de n'utiliser à long terme que la stratégie du levier, au détriment d'autres modes de séduction des publics internationaux. Mais cela n'empèche pas les cinéastes danois, August y compris, de tirer des conclusions stratégiques du succès de certains films comme Twist and Shout, Pelle le Conquérant et Les meilleures intentions. D'ailleurs, on pourrait affirmer que August joue avec le feu dans son dernier film, La maison aux esprits, précisément parce qu'il y renonce à la stratégie du levier qui est si importante dans ses autres films.

Département d'études anglaises

L'Université de McGill

Traduit de l'anglais par Stêphan D'Amour 